(C) [2010] IEEE. Reprinted, with permission, from [Jie Liang and Mao Lin Huang, Highlighting in Information Visualization: a Survey, Information Visualisation (IV), 2010 14th International Conference, 26-29 July 2010]. This material is posted here with permission of the IEEE. Such permission of the IEEE does not in any way imply IEEE endorsement of any of the University of Technology, Sydney's products or services. Internal or personal use of this material is permitted. However, permission to reprint/republish this material for advertising or promotional purposes or for creating new collective works for resale or redistribution must be obtained from the IEEE by writing to pubspermissions@ieee.org. By choosing to view this document, you agree to all provisions of the copyright laws protecting it 


\title{
Highlighting in Information Visualization: a Survey
}

\author{
Jie Liang and Mao Lin Huang \\ The University of Technology, Sydney, Australia \\ \{jiliang@it.uts.edu.au,maolin@it.uts.edu.au\}
}

\begin{abstract}
Highlighting was the basic viewing control mechanism in computer graphics and visualization to guide users' attention in reading diagrams, images, graphs and digital texts. As the rapid growth of theory and practice in information visualization, highlighting has extended its role that acts as not only a viewing control, but also an interaction control and a graphic recommendation mechanism in knowledge visualization and visual analytics. In this work, we attempt to give a formal summarization and classification of the existing highlighting methods and techniques that can be applied in Information Visualization, Visual Analytics and Knowledge Visualization. We propose a new three-layer model of highlighting. We discuss the responsibilities of each layer in the different stage of the visual information processing.
\end{abstract}

Keywords -- Highlighting, interactive visualization, information visualization, knowledge discovery, visual analytics

\section{Introduction}

Highlighting was one of the most common terms in computer graphics. However, there is little agreement on the understanding of highlighting for both nonprofessionals and professionals. For non-professionals, highlighting is either limited to the specific scope of color and lighting, as self-evidence of its name, or broadened to be the process of emphasizing information. In research, there is also a debate on highlighting definition for different domains of study.

In particular domain of information visualization, Becker and Cleveland in 1987 described highlighting as brushing special color to paint the object [3]; in 1999, Liston et al. illustrated highlighting as the process of emphasizing related sets of information, through visual annotation, within a view or across multiple views [14]; in 2003, MacEachren et al. referred highlighting as the indication method by using transient visual effects [15]; in 2004, Seo and Shneiderman suggested the term highlighting for the visual link across multiple views
[24]; in 2005, Ware and Borrow further defined it as an effective way with pre-attentive visual cues [32]. Recently, Ware and Borrow extended highlighting methods from static to dynamic approaches [33]. Nevertheless, these definitions all confined highlighting as the basic techniques for viewing only. Hence, it also implies that the understanding of highlighting in the literature of information visualization still remains in the lower level as viewing control mechanism.

However, the demanding of visual computing development has exceeded the capacity of current visualization tools for exploiting the meaningful information and maximizing the human's ability of interpreting. To response the challenges, this paper attempts to establish the highlighting as an essential component of visual computing to offer services for visual navigation and visual analytics.

Therefore, we define highlighting in three layers of different responsibilities and purposes

- In traditional view-based visualization, highlighting acts as the viewing control to attract user's attention into a portion of the visualization.

- In interactive visualization, highlighting functions as the navigation control mechanism to guide users progressively to reach the final target by visual interactions.

- In knowledge visualization and visual analytics, highlighting is further applied as part of artificial intelligence process to provide users with a set of graphical recommendations for the decision making.

Our new definition has gone beyond the limitation of traditional understanding of highlighting and moved on to the capability of speeding visual thinking and accelerating decision making performance. This profound change is intended not only to expand the boundary and fulfill the theory of highlighting, but also advance the potential development of highlighting in the future.

Therefore, the specific objectives of this paper are to refine and extend highlighting, and also to elaborate on the changing roles of highlighting from merely assisting with viewing, to interacting with information, participating directly in cognitive processes and finally suggesting for decision making. 


\section{The Conceptual Model of Highlighting}

Highlighting as independent component of visual computing should fundamentally be able to direct a new way to overcome the problem of information overload and reduce the process of human cognition, and meet challenges of collaborative analysis tasks. The new conceptual model we propose consists of three layers (Figure 1). The lowest layer is viewing control to solve the information overloading problem. The middle layer is interaction control that aims to ease the cognitive process for information seekers. The top layer is visual recommendation that mainly targets for decision makers in collaborative analysis tasks.

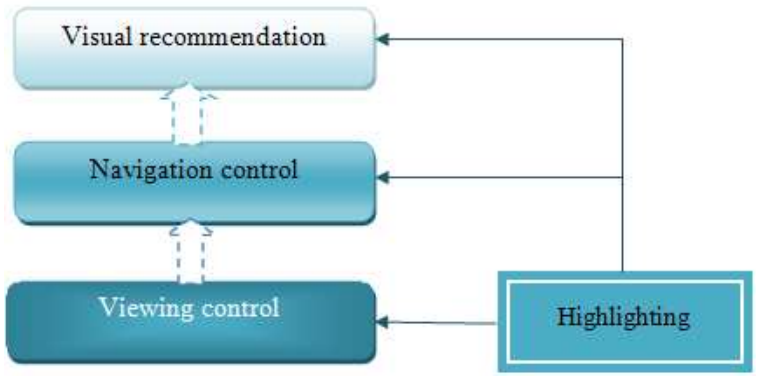

Figure 1: Conceptual model of highlighting

\subsection{Viewing Control for Information Overloading Problem}

Information overloading and complexity of information are the significant reasons for employing Highlighting. Due to the abundance, heterogeneity and continuous updating of the data, the visual representation of the data often generates visual clutters that add extra cognition overheads in viewing and understanding the information. Accordingly, the analysis processes have become increasingly time-consuming and complicated. Highlighting as a simple technique aims to reduce confusion, to minimize the learning curve and to increase accuracy and efficiency of understanding the information.

\subsection{Interaction Control for Information Retrieval}

In interactive visualization, highlighting has the capability to guide the information seeking process. For extensive and recursive cognitive thinking in finding the path towards the target view, highlighting should provide mechanisms allowing users progressively to reach their interested information in the most appropriate and comprehensive manner.

\subsection{Visual Recommendation for Decision Making}

In visual analytics and knowledge visualization, highlighting may attract decision makers' attention to a small portion of highly relevant information and knowledge that is directly beneficial for their decision making. This reduces significantly the time spent for decision makers on reading lower relevant information and knowledge.

Often analysts and decision makers are different people in commercial or research community. Refined highlighting in one work space can be the new solution for collaborative decision making challenge in projects particularly.

\section{The Elements of Highlighting}

In this section, we discuss the basic elements of highlighting, from which we can implement a variety of highlighting methods. Essentially, the "Differentiation" is the key to classify and implement highlighting; differentiation in light, shape, size, motion and presentation. The following table gives a summarization on the elements that have been used to design highlighting(Table 1):

\begin{tabular}{|c|c|c|}
\hline Differentiation & visual variables & series logic \\
\hline $\begin{array}{l}\text { Differentiation in } \\
\text { light }\end{array}$ & $\begin{array}{l}\text { Intensity } \\
\text { Frequency or } \\
\text { wavelength }\end{array}$ & $\begin{array}{l}\text { Light /medium /heavy } \\
\text { weak/strong }\end{array}$ \\
\hline $\begin{array}{l}\text { Differentiation in } \\
\text { size }\end{array}$ & $\begin{array}{l}\text { Area of object or } \\
\text { view } \\
\text { increase or decrease }\end{array}$ & Large/Medium/Small \\
\hline $\begin{array}{l}\text { Differentiation in } \\
\text { shape }\end{array}$ & $\begin{array}{l}2 \mathrm{D} \text { or } 3 \mathrm{D} \text { geometric } \\
\text { shapes } \\
\text { regular/irregular }\end{array}$ & Shape transformation \\
\hline $\begin{array}{l}\text { Differentiation in } \\
\text { motion }\end{array}$ & $\begin{array}{l}\text { speed, acceleration, } \\
\text { displacement, and } \\
\text { time }\end{array}$ & $\begin{array}{l}\text { motionless/relevant } \\
\text { motion/absolute } \\
\text { motion }\end{array}$ \\
\hline $\begin{array}{l}\text { Differentiation in } \\
\text { presentation }\end{array}$ & $\begin{array}{l}\text { style-based } \\
\text { Line-based }\end{array}$ & $\begin{array}{l}\text { Abstract/details } \\
\text { focus/outlier/boundary }\end{array}$ \\
\hline
\end{tabular}

Table 1: The elements of highlighting

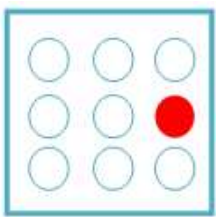

(a)

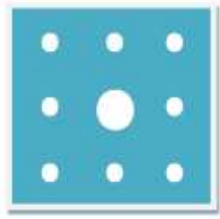

(d)

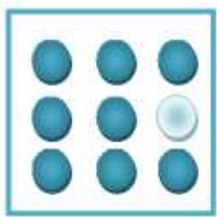

(b)

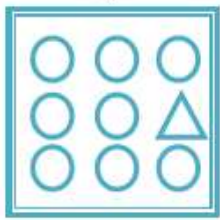

(e)

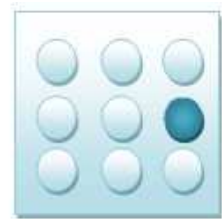

(c)

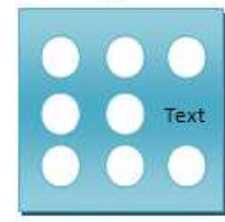

(f)
Figure 2: Simplified Samples of Differentiation in graphic elements (a) Color (b) Light contrast (c) Transparency (d) Size (e) Shape (f) Presentation 


\section{The Classification of Highlighting}

\subsection{Viewing Control Layer}

Viewing control process is based on pre-defined algorithms. (Figure 3) The visualization defines the user's "interested" portion of the information and highlights the corresponding portion of the view to attract user's attention. In this layer, most elements are commonly used to implement the highlighting, such as color, light, size, shape etc.

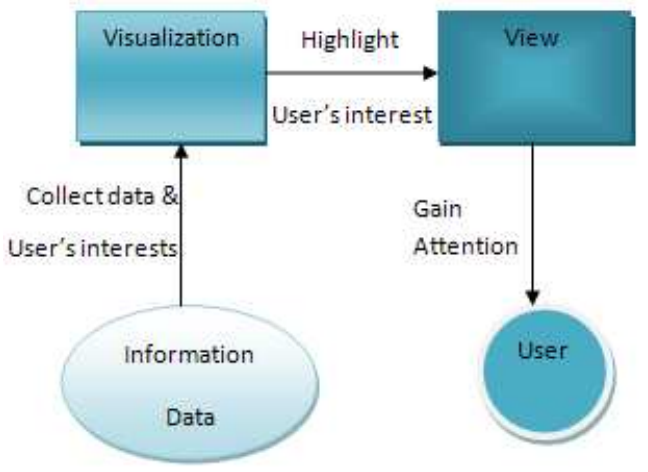

Figure 3: The framework of viewing control

\subsubsection{Differentiation in light}

4.1.1.1 Color-based: Different range of spectrum, displays various color groups for highlighting. The color difference and level of saturation can be used to distinguish the highlighted objects, depending on the required details of views in specific visualization environment. For example, growth matrix in financial data visualization [11], with color and color saturation highlighting techniques (Figure 2a) becomes possible to show the performance of a fund in all time intervals over a time period of 14 years (about 11.000 intervals).

4.1.1.2 Light Contrast-based: Contrast also can be used to visually separate highlighted objects from background information. (Figure 2b) Modification relies on measurements to control either how sharp certain objects appear, or how much blur should apply to background information to make the highlighted objects appear more obvious. In geo-visualization, it is sometime called "Depth of field" [22]. For example, visualization tool of Kosara et. Al., adopted semantic depth of field (SDOF) method [13] and the user studies have proved its usefulness.

4.1.1.3 Transparency can be used as a transient highlighting technique to dissolve the context around the object of interests. (Figure 2c) The alpha level of objects can be set to render the focused object in the display, but this potential method has not been evaluated. Nguyen \& Huang's work in layering and transparency [17] provides initial reference for further implementation, but it is still a challenge to determine the appropriate level of transparency for displays that have been colored with light-to-dark color schemes.
The differentiation in lighting can be operated into one, two, three or even multiple dimensions visualizations based on different applications. Parallel coordinate visualization has successfully used highlighting to control the viewing. It displays the focused poly-lines within a thin region. Alternatively, color and light highlighting are used to select data in specific scope of dimensions [26]. XmdvTool [16] created by Martin and Ward progressed the function to highlight a series of data of high dimensions in a "brushed tunnel" (Figure 4). Siirtola and Raiha further advanced this solution into a new version of parallel coordinate explorer interface [26]. They rearranged data into multiple layers of highlighting by adopting differentiation in light(Figure 5).

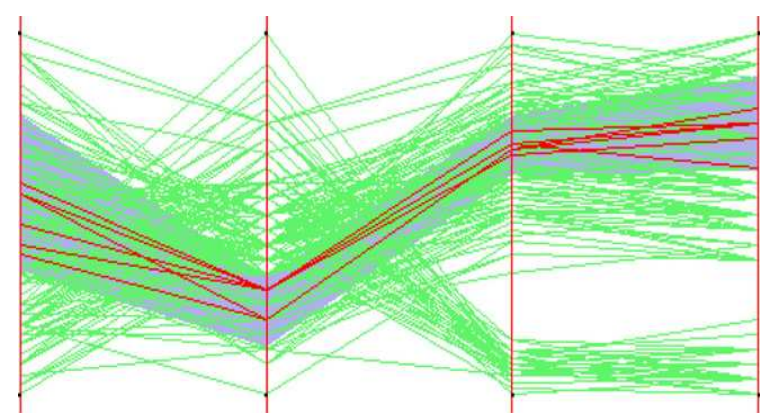

Figure 4: Highlighting in Multidimensional data. The region brushed gray color underneath the poly-lines is the "tunnel" which determines the range of data selection in multiple dimensions. [16]

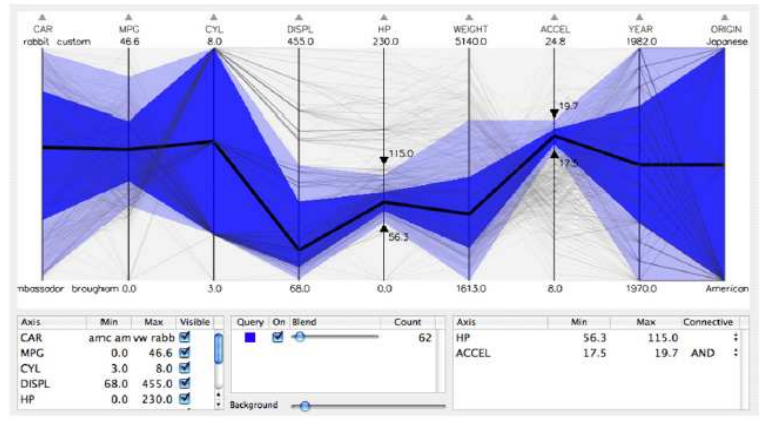

Figure 5: A poly line abstraction based on the box-andwhiskers plot in two tunnels (grey region and blue region) [26]

\subsubsection{Differentiation in size}

Size-based highlighting includes the diversification of dimension changes. (Figure 2d) Sensible strategy of modification also remains discovering. It is specially applied to the situation when color or light based methods fail to deliver the ideal outcomes, especially for color-blinds who have troubles to distinguish color and light. Taking example of text annotation, the color and light variation seems the simplest solution for the presentation of overlapping digital annotation from different authors, but only under the strict restriction of 
Authors' number. Beyond three authors, the visual annotation presentation would look like a confusing color palette. We could hardly identify or compare the annotations from different authors in digital applications [30]. In this situation, the size of text annotation is likely another indicator to go beyond three authors for annotation comparison visualization.

\subsubsection{Differentiation in shape}

Similarly, shape has been applied into existing practices (Figure 2e). Taking email visual organization as an instance, in order to expand details of email indicators, Bernald Kerr [12] successfully adopted color highlighting of different scheme and moved on to the indicator of shape. In attribute highlighting, Kerr naturally used color desaturation to rate the importance of emails, and employed different shapes of circle, solid circle, hollow circle to present the importance of people.

\subsubsection{Differentiation in presentation}

4.1.4.1 Style-based: The idea in style reduction or addition is inspired by highlighting data objects in geovisualizations [21]. Style for highlighting especially works well with visual representations that are designed with multiple graphical elements. (Figure 2f) We may alter styles by reducing or adding or changing, in outlining, labeling, and other graphical elements. For example, in internet hyperbolic tree visualization [8], German home and French home page attracts users' attention with added flag presentation.

4.1.4.2 Line-based method leans to be used for indicating relationship between positions of data or objects, for example, leader lines [20] and contouring line [21] adopted from map design, also give differentiation for the object from neighborhood. The challenge this highlighting method involves connecting lines to linear representations as shown in the parallel or multiple views. Line based highlighting can also be drawn in variations of color, width, and stroke style. However, the contouring method has not been adopted in prior work as a highlighting style yet.

\subsubsection{Differentiation in Motion}

Microsoft PowerPoint elicits the method of motion highlighting in its customization of presentation slides. It has four categories, by entrance, by exit, by emphasis, and by motion path. The entrance and exit highlighting include show and hide function and other dynamic effects. The emphasis positioning may include spin, arrow, circular [31], jolt [31], radiating and flash or combination. For example, in our previous work of trading network visualization [10], a flashing circle highlighting over a suspected focus can be further implemented to identify a suspected behavior of domination of the stock price. In the motion path highlighting, the object or view may move in straightline or along curve according to certain shapes. Bartram's usability study [2] proved that motion highlighting is much more effective than color or shape change in signaling a change to a data glyph presented on the screen. He also further supported his assumption that motion highlighting technique is promising solution for separating a group of objects enabling a rapid visual search on the groups.

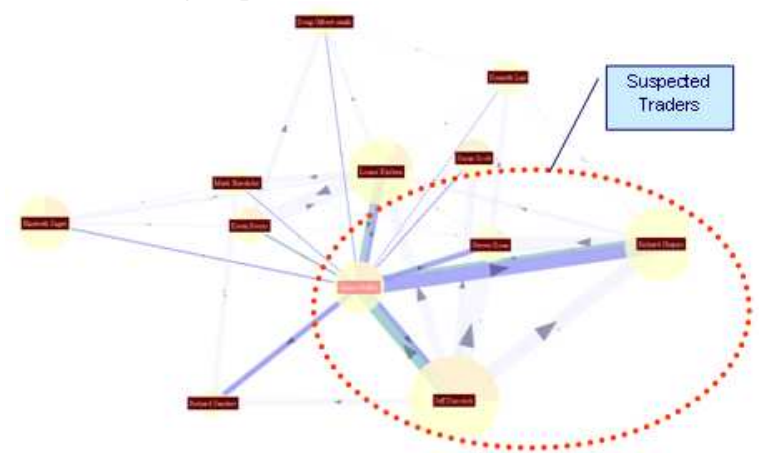

Figure 6: Motion highlighting: A flashing circle outlines a suspected behavior of domination of the stock price in Huang and Liang and Nguyen's demo [10]

\subsection{Interaction Control Layer}

In interaction control, the highlighting is applied in navigation process that guides users to progressively refine their focus views to reach the final target view. The navigation process may also be the process of data retrieval (see Figure 7). This highlighting layer may apply differentiation in most elements, particularly size of region.

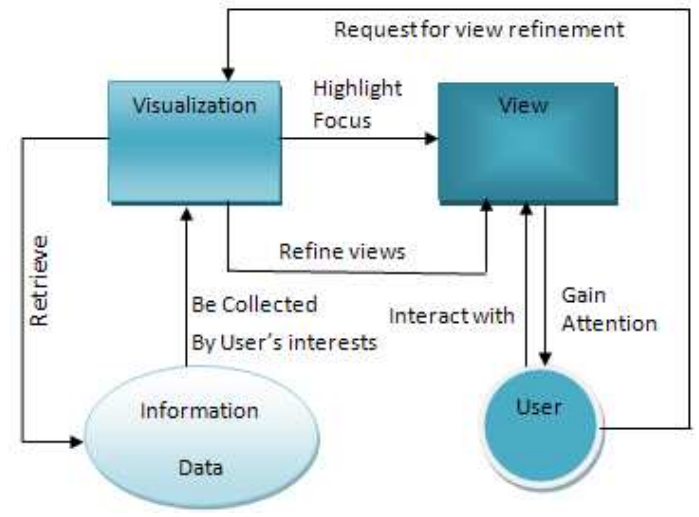

\section{Figure 7: the framework of Interaction Control}

\subsubsection{Differentiation in Size of Region}

Most view-based highlighting takes advantage of differentiation in size and shape to provide focus and background information based on the details degree of user requirements. With the support of available information, users may modify view transformation and browse and switch the data by different granularity with highlighting indications.

4.2.1.1 One view: In partial view highlighting, drilling-down + semantic zooming adopting differentiation of size and shape is the most commonly used interactive navigation technique, for example, fisheye + zooming [25], power finger, and view 
distortion. This quick and simple navigation scheme is widely established in the current operation systems, web researching and many common applications. Ben Bederson, director of the Human-Computer Interaction Lab, from University of Maryland created Fisheye Menus [4] with size differentiation highlighting, for the application of linear lists. The interactive Map of Washington D.C, also is applied with this primary highlighting technique, to increases size of the station "Metro centre" and transforms the shape into the fish eye in the navigating (Figure 8).

4.2.1.2 Two views: In coordinated views, Focus and context view is another existing method relying on element of size and shape. It provides users with a detailed view of a focused sub-graph and overall graph to maintain user orientation. Typical techniques in focus + context navigation scheme include sunburst [28], and information slices [1].

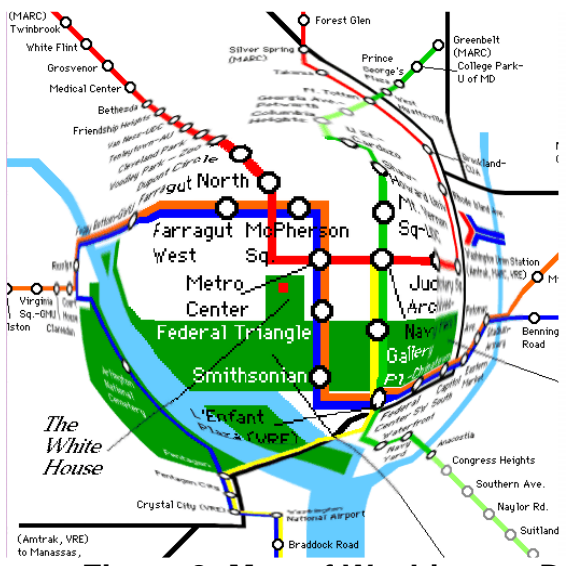

Figure 8: Map of Washington D.C. [35]

4.2.1.3 In different views, we also include merging views, adopted from "overlay" technique in construction planning. [14] This new highlighting method merges the views from different team members into one view. To review the project, the team could focus on comparing and evaluating the important information on the "merged" view. This method implements following types of overlaying actions, overlaying document to document of same type, objects to document of same type and document to document of different type and objects to document of different type by certain mechanisms.

4.2.1.4 In Multiple views, visual highlighting techniques have been largely unexplored in research. [21] Our prior work in Full-context views visualization with chains of full history is proposed to be one of highlighting techniques in multiple views by increasing the context of an entity collection step by step (Figure 9). Full-context view Visualization has successfully solved the conflict between the goal of providing structure and the goal of visualization detail context, by integrating the presentation of structure with the presentation of context [10]. In the figure 9, referring to the historical times from multiple context views, and enlarged focus views, analysts could match this significant event to the suspected trading patterns which had frequently occurred in history and accordingly analysts might be able to identify the actual plans and predict the next action.

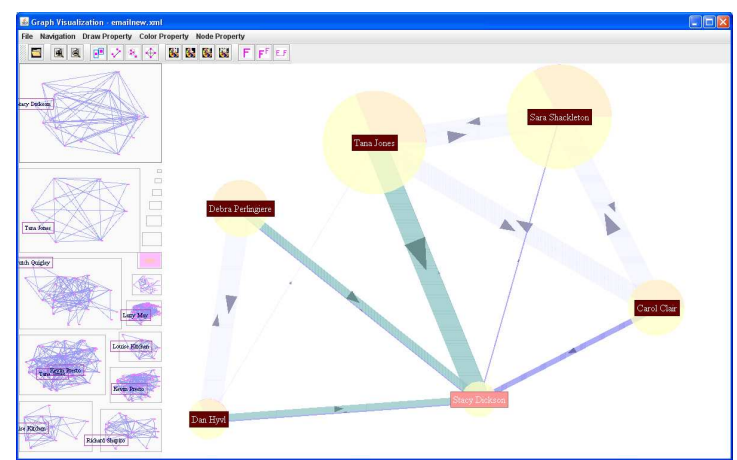

Figure 9: Full context view visualization [10]

\subsubsection{Differentiation in light}

The previous work of context and focus visualization by Huang and Nguyen [17], has proved that if transparency level is carefully selected, it can be designed to guide users in navigation as a highlighting method. In Figure 10, while the background of structure fades, the view of "Applets" sub-structure is standing out of the whole data structure and the size of the focus view is also enlarged in the space filling presentation

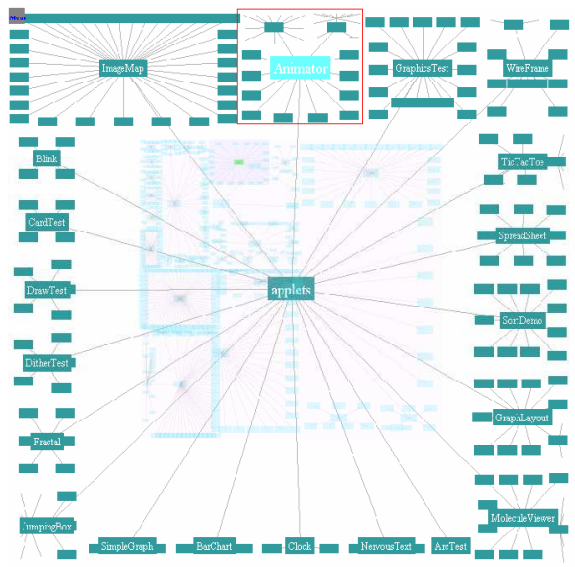

Figure 10: Highlighting with differentiation in Transparency: The data structure stands out of the semi-transparency layers. [17]

\subsection{Visual Recommendation}

In knowledge visualization and visual analytics the role of highlighting can be further extended as part of the knowledge discovery process to provide users with a set of graphical recommendations for their decision making. In knowledge discovery, a large volume of the raw data is pre-processed by analytical algorithms, such as data mining or feature selection algorithms. These algorithms are generated by domain experts or statistical methods based on empirical or mathematical theory support at the background. The outcome knowledge of the analysis is used for facilitating the decision making process. 


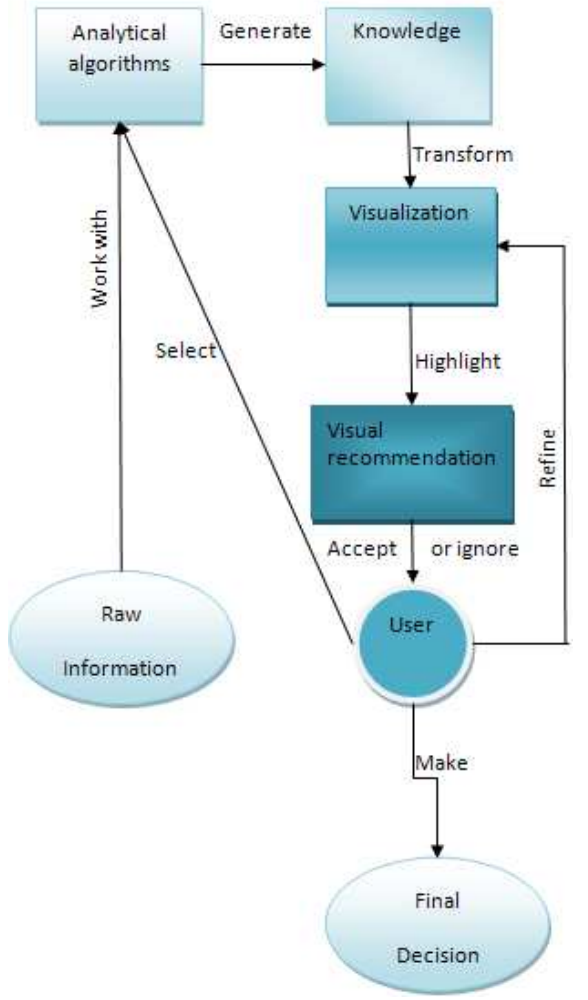

Figure 11: The framework of Visual Recommendation

However, in many cases, the textual or tabular representations of the knowledge are hard to be understood by decision makers, especially the relational structures among knowledge. Therefore, the modern visualization technologies allow decision makers to take advantages of human vision to visually interpret the knowledge. However, for a particular decision maker, he or she may only needs a specific segment of the knowledge, rather than a complete set of knowledge displayed in the visualization for the decision making. Therefore, we "highlight" particular segments of the knowledge in the visualization as visual recommendations, which we believe are the most beneficial for a particular decision making process. In the process, users have choices to either accept or ignore the visual suggestions. When visual results are unsatisfied, users are allowed to refine the visualization with different highlighting mechanism. Consequently, this visual recommendation process can be iterative. In brief, visual recommendation could not only suggest a shortcut for user to reach the knowledge he or she pursue for decision making, but also enhance the knowledge discovery experience for users.

For example, Visual thesaurus [29] powered by think map uses intelligence to suggest the words relating to or synonyms in a given language. It adopts color and size and shape highlighting as viewing aid. Rollover motion highlighting along with "back and forward" button allows users to interact and navigate in the maps of words. To assist learning the knowledge in thesaurus, it utilizes the visual recommendation process and highlights the suggested words and meaning based on system's definition of the term and allows users to act with suggestions. In this way, users can either narrow or broaden the meaning of term based his or her own understanding and discover the knowledge. In figure 12, the user looked up the word "highlighting" and the think map highlighted six suggestions within the thesaurus. In succession, the user clicked on the one of the suggestions. The think map centralized and magnified the word "spotlight" and displayed another series of suggestions. (Figure 12)

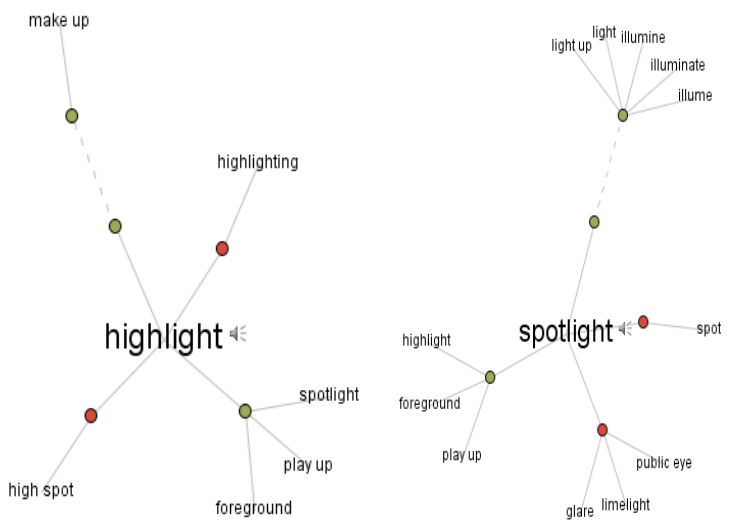

Figure 12: Visual Thesaurus [29]

Left: when user looked up a term" highlight" in visual Thesaurus: Right: When user clicked on spotlight among previous suggestions

\section{Future Work \& Conclusion}

Up to now the term of highlighting has not been defined clearly. Most designers ignored the formal explanation and systematical theory. In most cases, highlighting is still taken for grant as the use of color and light. As a result, highlighting has been limited to basic viewing aid in most applications.

As closely relating to visual representation and Interaction, highlighting is required to raise up to higher level of information visualization process. Therefore, this paper has attempted to define and classify the existing highlighting techniques. The highlighting theory should be explored further in our future work. Particularly, we should put effort in investigation of the combination of multiple highlighting techniques to satisfy the needs in some new applications. It is required to have the highest standards which should have the real look working with visualization and the real feel working with interaction.

Highlighting covers many disciplines and areas of experiences, like science of cognition, user physiology, aesthetics, human vision study, visualization and visual analytics. The strong theory base is the ground for the evolution of highlighting. To ensure highlighting as 
lynchpin in visualization and interaction, we should continue to fulfill the theory development and extend practices of highlighting and advance the user-center evaluation for highlighting in each layer of services.

\section{References}

[1] Andrew, K. \&Heidegger, H. 1998, 'Information Slices: Visualizing and exploring Large Hierarchies using Cascading, Semi- Circular Discs', Proceedings of IEEE Symposium on Information Visualziation,1998, IEEE, pp.9-12.

[2] Bartram, L. \& Ware C. 2002, 'Filtering and brushing with motion.' Information Visualization 1(1): 66-79.

[3] Becker, R. \& Cleveland, W. 1987, 'Brushing scatterplots', Technimetrics, vol. 29, no. 2, pp. 127-142.

[4] Bederson, B. 2000.' Fisheye menus', ACM.

[5] Czerwinski, M., Dumais S., et al. 1999, 'Visualizing implicit queries for information management and retrieval', ACM.

[6] Gravity Investment, Inc. http://www.gravityinvestments. com/portfoliopics.htm.

[7] Gross, J. and Hayne H. 1999, 'Young children's recognition and description of their own and others' drawings.' Developmental Science 2(4): 476-489.

[8] Hyper Tree retrieved Feb $20 \quad 2010$ from http://Hypertree .sourceforge.net/.

[9] Huang, M. \& Nguyen, Q. 2008, 'Context Visualization for Visual Data Mining', Visual Data Mining, pp. 248-263.

[10] Huang, M., Liang, J. \& Nguyen, Q. 2009, 'A Visualization Approach for Frauds Detection in Financial Market', IEEE Computer Society, pp. 197-202.

[11] Keim, D.A., Mansmann, F., Schneidewind, J. \& Ziegler, H. 2006, 'Challenges in Visual Data Analysis', Information Visualization, Vol. July, pp. 9-16.

[12] Kerr, B. 2003, 'Thread arcs: An email thread visualization', Citeseer.

[13] Kosara, R., S. Miksch, et al. 2001, 'Semantic depth of field', Citeseer.

[14] Liston, K., Fischer, M. et al. 2000, 'Designing and evaluating visualization techniques for construction planning.' Computing in Civil and Building Engineering: 1293-1300.

[15] MacEachren, A., F. Hardisty, et al. (2003). 'Supporting visual analysis of federal geospatial statistics.' Communications of the ACM 46(1): 60 .

[16] Martin, A. \& Ward, M. 1995, 'High dimensional brushing for interactive exploration of multivariate data', IEEE Computer Society, p. 271.

[17] Nguyen, Q.V. \& Huang, M,L. 2004, 'A Focus + Context Visualization Technique Using Semi-Transparency', Proceedings of the Fourth International Conference on Computer and information Technology, IEEE Computer Society, pp.101-108.

[18] Nguyen, Q. \& Huang, M. 2005, ' EncCon: an approach to constructing interactive visualization of large hierarchical data', Information Visualization, vol. 4, no. 1, pp. 1-21.

[19] Plumlee, M. \& Ware C. 2006). 'Zooming versus multiple window interfaces: Cognitive costs of visual comparisons.' ACM Transactions on Computer-Human Interaction (TOCHI) 13(2): 209.

[20] RINA Systems, Inc. Retrieved May 52008 from http://www.rinafinancial.com/.

[21] Robinson, A. \& Center, G. 2006, 'Highlighting techniques to support geovisualization', Citeseer.
[22] Robinson, A. 2009, 'Visual highlighting methods for geovisualization',Citeseer.

[23] Santini, S., Jain, R. et al. 1999, 'Social and Content-based Approach for Visual Recommendation of Web Graphics', Citeseer.

[24] Seo, J. and Shneiderman B. 2005, 'A rank-by-feature framework for interactive exploration of multidimensional data.' Information Visualization 4(2): 96-113.

[25] Shi, K., Irani, P. \& Li, B. 2005, 'An Evaluation of Content Browsing Techniques for Hierarchical SpaceFilling Visualizations', Proceedings of IEEE Symposium on Information Visualization, 2005, IEEE, pp. 81-88.

[26] Siirtola, H. \& KJ, R. 2006, 'Interacting with parallel coordinates', interacting with Computers, vol. 18 , no. 6 , pp. 1278-1309.

[27] SmartMoney, Inc. http://www.smartmoney.com.

[28] Stasko, J. \& Zhang, E. 2000, 'Focus + Contest Display and Navigation Techniques for Enhancing Radial, SpaceFilling Hierarchy Visualizations', Proceedings of IEEE Symposium on Information Visualization, 2000, IEEE, pp. 57.

[29] ThinkMap Visual Thesaurus, http://www.visualhesaurus. Com.

[30] Villarroel, M., P. de la Fuente, et al. (2006). 'Visualizing Shared Highlighting Annotations.' HCI related papers of Interacci 2004: 195

[31] Ware, C. \& Bobrow R. 2004, 'Motion to support rapid interactive queries on node--link diagrams', ACM Transactions on Applied Perception (TAP) 1(1): 3-18.

[32] Ware, C. \& Bobrow, R. 2005, 'Supporting visual queries on medium-sized node-link diagrams', Information Visualization, vol. 4, no. 1, pp. 49-58.

[33] Ware, C., Gilman, A. et al. 2007, 'Visual Thinking with an Interactive Social Network Diagram'.ACM

[34] Wexelblat, A. \& Maes P. 1999, 'Footprints: history-rich tools for information foraging', ACM New York, NY, USA.

[35] WashingtonD.C.InteractiveMap,www.cs.umd.edu/class /fall2002/cmsc838s/tichi /fisheye.Html.

[36] Wong, P., Foote, H. et al. 2006, 'Graph signatures for visual analytics.' IEEE Transactions on Visualization and Computer Graphics 12(6): 1399-1413. 